Trauma Berufskrankh 2010 - 12[Suppl 2]:153-156 DOI 10.1007/s10039-010-1647-2

Online publiziert: 27. Mai 2010

(c) Springer-Verlag 2010

\author{
V. Bühren \\ BG-Unfallklinik Murnau
}

\section{Heilverfahren der DGUV}

\section{Heilverfahren aus der Sicht der Fachgesellschaften}

Die treibenden Prozesse für die Entwicklung der Heilverfahren in der gesetzlichen Unfallversicherung lassen sich in 3 Punkten kumulieren.

\section{Veränderungen im Vertragsarzt-} recht. Die Veränderungen in der ambulanten Versorgung wurden im Jahr 2007 mit dem Vertragsarztrechtveränderungsgesetz initiiert, das wesentliche Umstrukturierungen im ambulanten Versorgungsbereich zur Folge hat. Der klassische Durchgangsarzt (D-Arzt) in eigener Praxis als Einzelperson wird abgelöst durch Gemeinschaftspraxen, teilweise mit mehreren Standorten, dabei ergänzend mit Ärzten in Anstellungsverhältnissen, verbunden auch mit einer operativen Tätigkeit in auswärtigen ambulanten Operationszentren, in Krankenhäusern als Belegund Konsiliarärzte. Dieser veränderten Versorgung müssen naturgemäß auch die Regelungen des Durchgangsarztverfahrens zum ambulanten Bereich Rechnung tragen.

Veränderungen in der Aus- und Weiterbildung. $\mathrm{Ab} 2004$ sind das ursprüngliche Fachgebiet Orthopädie sowie der Schwerpunkt Unfallchirurgie aus dem Fachgebiet Chirurgie zu einem neuen Fachgebiet Orthopädie und Unfallchirurgie unter dem gemeinsamen Hauptfach Chirurgie vereint. Entstanden ist eine neue Facharztbezeichnung Orthopädie und Unfallchirurgie mit deutlich anderen Anforderungen und einem veränderten Qualifikationsprofil, was wesentliche Auswirkungen auf die Anforderungen zur Teilnahme als Durchgangsarzt an den Heilverfahren hat.
Veränderungen auf Kostenträgerebene. Im Jahr 2008 begannen, wesentlich durch die Vorgaben des Unfallversichungsmodernisierungsgesetzes hervorgerufen, Umstrukturierungen im Bereich der gesetzlichen Unfallversicherung, die neben der dramatischen Verringerung der Zahl der gewerblichen Berufsgenossenschaften gleichzeitig in einem Zusammenschluss mit den ebenfalls in der Zahl reduzierten Unfallkassen resultierten. Unter dem Zusammenschluss als Deutsche Gesetzliche Unfallversicherung (DGUV) mit Sitz in Berlin ist zugleich eine Zentralisierung in der prinzipiellen Regelung für die Heilverfahren eingetreten. Auch im neuen Gesetzgebungsverfahren ist es beim alleinigen Organisationsrecht der Unfallversicherungsvertreter nach SGB (Sozialgesetzbuch) VII $\$ 34$ geblieben, der eine Steuerung des Heilverfahrens über die Zulassung von Ärzten und Krankenhäusern entsprechend den Anforderungen für das Heilverfahren zulässt.

Aus Sicht der Fachgesellschaften bestand die Notwendigkeit, in die Neuregelungen die Aspekte der veränderten Weiterbildungsordnung, v. a. aber auch die medizinischen Fortschritte in der Akutversorgung und in der rekonstruktiven Orthopädie in die Heilverfahren einzubringen. Einen wesentlichen Einfluss hat die Entwicklung des Traumanetzwerk ${ }^{\mathrm{D}}$ der Deutschen Gesellschaft für Unfallchirurgie (DGU), mit dem sich für die Strukturen zwischen den bestehenden, am Verletzungsartenverfahren beteiligten Kliniken erhebliche Synergieeffekte ergeben.

\section{Künftige persönliche Anforderungen an den Durchgangsarzt}

Grundlage der bisherigen Zulassung zum Durchgangsarzt war der Facharzt für Chirurgie mit einer danach erfolgenden Zusatz- oder Schwerpunktbezeichnung in der Speziellen Unfallchirurgie. Vor 1993 dauerte die Facharztweiterbildung 6 Jahre, mit der Weiterbildungsordnung 1993 wurde sie auf 5 Jahre verkürzt. Die frühere $\mathrm{Zu}$ satzbezeichnung erforderte eine weitere 2jährige Qualifikation, die Schwerpunktbezeichnung zusätzlich 3 Jahre Tätigkeit auf Facharztniveau. Die Durchgangsarztqualifizierung dauerte somit formal 8 Jahre, durch die mögliche Anrechnung 1 Jahres aus der Spezialisierung in die Facharztweiterbildung konnte sie auch in 7 Jahren erreicht werden. Die Weiterbildungsinhalte umfassten große Teile der so genannten allgemeinen Chirurgie, darin ohne besondere Definition eingebundene unfallchirurgische Inhalte, jeweils ergänzt durch die 2- bis 3-jährige spezielle unfallchirurgische Weiterbildung.

Mit Einführung des Fachgebiete Orthopädie und Unfallchirurgie durch die Weiterbildungsordnung 2004 wurde der Facharzt quasi in 3 Abschnitte strukturiert:

- In den ersten 2 Jahren erfolgt wie in allen chirurgischen Fachgebieten die Weiterbildung im so genannten „common trunk“ mit Schwerpunkten in der Stationsführung, in der ambulanten Versorgung und auf der Intensivstation. 


\section{Heilverfahren der DGUV}

D-Arzt künftig "Basis”

FA Chirurgie + 1 Jahr VAV-UCh + Kurse

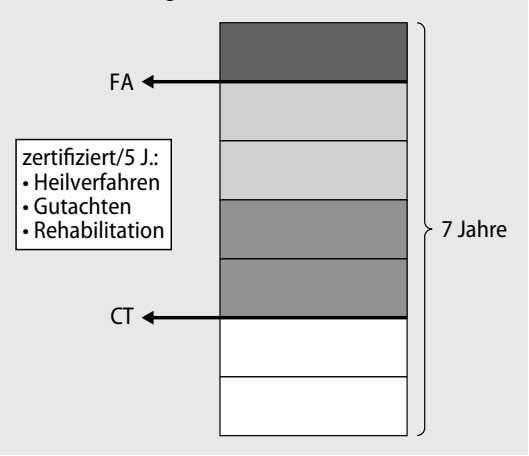

Abb. $1 \Delta$ Niedergelassener Basisdurchgangsarzt, Ausbildungsanforderungen: schwarz 1 Jahr VAV, hellgrau Orthopädie, dunkelgrau Unfallchirurgie, weiß CT, Anzahl der Balken Jahre, CT "common trunk", FA Facharzt, UCh Unfallchirurgie, VAVVerletzungsartenverfahren

- An den ersten Abschnitt schließt sich ein 4-jähriger Block an, der für die Orthopädie und Unfallchirurgie mit jeweils 2 Jahren im jeweiligen Fachgebiet anzusetzen ist. Hierzu ist festzuhalten, dass der Orthopäde und Unfallchirurg mit Facharztabschluss 2 Jahre konzentrierte Unfallchirurgie absolviert, ergänzt durch eingehende Erfahrungen im orthopädischen $\mathrm{Ne}$ benfach. Somit ist von vornherein eine höhere spezialisierte Qualifikation anzunehmen, als sie die frühere Fachartzweiterbildung Chirurgie regelhaft vermittelte.

- Auch das Fachgebiet Orthopädie und Unfallchirurgie nutzt die spezielle $\mathrm{Zu}$ satzbezeichnung Spezielle Unfallchirurgie. Hierfür werden in Analogie zur früheren Schwerpunktbezeichnung 3 Jahre veranschlagt.

Somit ergibt sich ein Zeitbedarf für eine unfallchirurgisch hochwertige Qualifikation von 9 Jahren, durch Anrechnung 1 Jahres ist eine Verkürzung auf 8 Jahre erreichbar.

Im Vergleich des Unfallchirurgen alter Prägung mit dem spezialisierten Unfallchirurgen nach WO (Weiterbildungsordnung) 2004 ist festzustellen, dass der Qualifikationsgang nunmehr um 1 Jahr verlängert ist und deutlich mehr Inhalte in der Unfallchirurgie vermittelt. Auf der anderen Seite bleibt festzustellen, dass die Inhalte der Allgemeinchirurgie für den Unfallchirurgen neuer Prägung deutlich re-
D-Arzt künftig Basis + operativ + VAV FA Ortho./UCh + Spez. UCh + Kurse

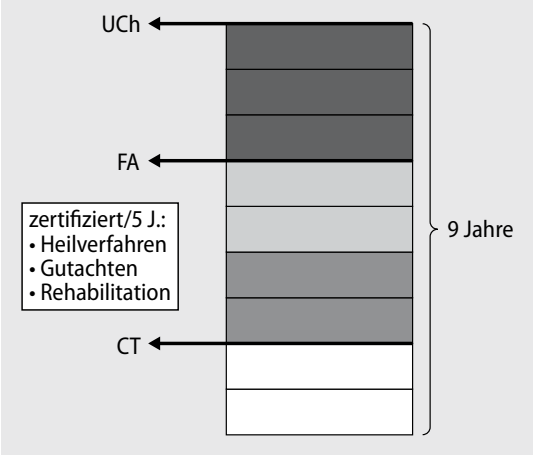

Abb. $2 \Delta$ D-Arzt mit operativer Tätigkeit, Ausbildungsanforderungen, schwarz 3-jährige spezielle unfallchirurgische Weiterbildung, hellgrau Orthopädie, dunkelgrau Unfallchirurgie, weiß CT, Anzahl der Balken Jahre, $C T_{\text {" }}$ common trunk", $F A$ Facharzt, Ortho. Orthopädie, Spez. spezielle, UCh Unfallchirurgie, VAVVerletzungsartenverfahren

duziert wurden, was naturgemäß Auswirkungen auf die Kompetenz für die Versorgung des Viszeraltraumas haben muss.

In der eingehenden Diskussion der Fachgesellschaften mit der DGUV kam zum Ausdruck, dass ein 9-jähriger Weiterbildungsgang mit dem Erwerb umfassender operativer Fähigkeiten für eine Niederlassung als Orthopäde und Unfallchirurg mit Beteilung am Durchgangsarztverfahren nicht zielführend sein kann. Gerade in der Niederlassung bestehen eine Reihe deutlich anderer Qualifikationsmerkmale, die in einer weitgehend operativ ausgerichteten Weiterbildung nur unzureichend berücksichtigt werden. In der Konsequenz dieses Gedankenganges wurde das Durchgangsarztverfahren auf ein modulares System umgestellt, das in einer Dreistufigkeit den Basisdurchgangsarzt in der Niederlassung, den operativ tätigen Durchgangsarzt vorzugsweise am Krankenhaus, aber auch an großen ambulanten Zentren, und den Durchgangsarzt am für das Verletzungsartenverfahren (VAV) zugelassenen Haus beschreibt.

\section{Niedergelassener Durchgangsarzt}

Seine Aufgabe ist die umfassende Betreuung der Versicherten, aber ohne persönliche Durchführung spezieller operativer Verfahren.

Gefordert werden der Facharzt für Orthopädie und Unfallchirurgie sowie ein weiteres qualifizierendes Jahr in der Unfallchirurgie, ergänzt durch Kurse zu den
D-Arzt künftig Basis + operativ + VAV FA Ortho./UCh + Spez. UCh + 3 Jahre VAV + Kurse

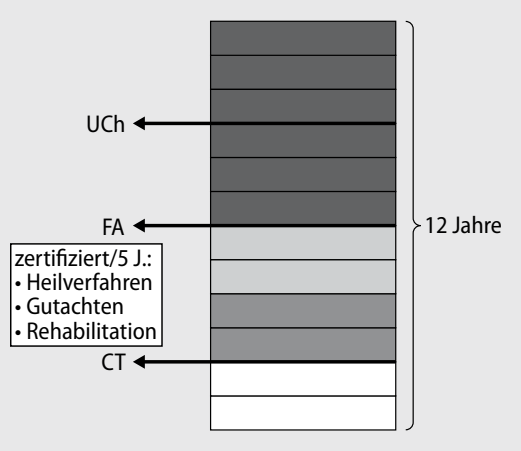

Abb. $3 \Delta$ D-Arzt im VAV, Ausbildungsanforderungen, schwarz 3-jährige spezielle unfallchirurgische Weiterbildung, hellgrau Orthopädie, dunkelgrau Unfallchirurgie, weiß CT, Anzahl der Balken Jahre, $C T_{\text {" }}$ common trunk", FA Facharzt, Ortho. Orthopädie, Spez. spezielle, UCh Unfallchirurgie, VAVVerletzungsartenverfahren

relevanten Inhalten der Patientensteuerung. Das zusätzliche unfallchirurgische Jahr muss an einem zum Verletzungsartenverfahren zugelassenen Haus und innerhalb diesem in einem für das Verfahren relevanten Bereich, am besten in der DA-Ambulanz (D-Arzt-Ambulanz), abgeleistet werden. Die Tätigkeit ist durch ein qualifizierendes Zeugnis zum Durchgangsarzt zu bestätigen. Die zusätzlichen Kurse sind zunächst als üblicher Einführungskurs für den Durchgangsarzt nachzuweisen, ergänzt durch zertifizierte Kurse in der Gutachtenerstellung und zu den Verfahren und Organisationsformen der Rehabilitation (• Abb. 1).

\section{Durchgangsarzt mit operativer Tätigkeit}

Die Anforderungen bauen praktisch modular auf den Basisanforderungen auf. Allerdings wird eine 3-jährige spezielle unfallchirurgische Weiterbildung gefordert, die mit dem Erwerb der Zusatzbezeichnung Spezielle Unfallchirurgie abschließt. Identisch wird die Absolvierung der Kurse wie im Basismodul gefordert.

Letztendlich entsprechen die Anforderungen für diesen Typ des Durchgangsarztes der bisherigen Regelung für die Absolventen nach Weiterbildungsordnung 2004. Bevorzugter Arbeitsplatz für diese Durchgangsärzte werden der stationäre Bereich und somit die zum Durchgangsarztverfahren zugelassenen Krankenhäuser sein. Denkbar sind jedoch auch die 


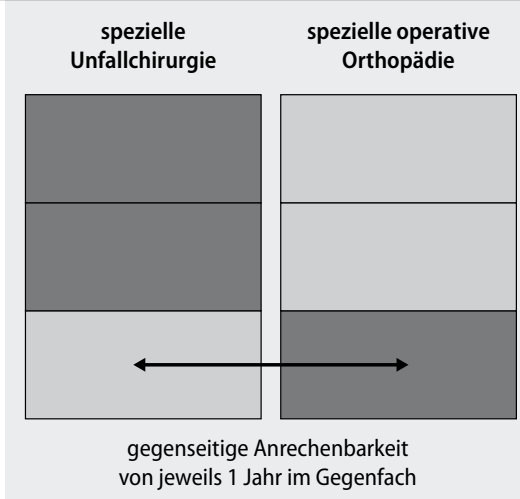

Abb. $4 \Delta$ Anrechenbarkeit der Weiterbildungen für Spezielle Unfallchirurgie bzw. Spezielle operative Orthopädie

Tätigkeit an entsprechend ausgerichteten großen ambulanten Zentren, ggf. auch die beleg- oder konsiliarärztliche Tätigkeit an Krankenhäusern (• Abb. 2).

\section{Durchgangsarzt im Verletzungsartenverfahren}

Nach Erreichung der Voraussetzungen für den operativen Durchgangsarzt wird eine mindestens weitere 3 -jährige Tätigkeit in verantwortlicher Stellung an einem zum Verletzungsartenverfahren zugelassen Haus gefordert. Der Zeitbedarf für diese Qualifikation beträgt somit insgesamt 12 Jahre und entspricht damit den bisherigen Regelungen, für die im Übrigen guten Erfahrungen und eine durchgehende Akzeptanz bestehen ( Abb. 3).

\section{Spezielle Unfallchirurgie und Spezielle operative Orthopädie}

Diskutiert werden auch die Synergismen zwischen den Zusatzbezeichnungen für die Spezielle Unfallchirurgie sowie für die bisher schon definierte und jetzt in die WO 2004 aufgenommene Spezielle operative Orthopädie. Angesichts einer deutlichen Überlappung der inhaltlichen Anforderungen scheint es durchaus denkbar, dass jeweils 1 Jahr aus dem jeweiligen Weiterbildungsgang vice versa anerkannt werden kann (• Abb.4). Die Art und Weise der Anerkennung ist Sache der Landesärztekammern, entweder über eine prinzipielle Regelung in der Weiterbildungsordnung oder aber über die Anerkennung als gleichwertiger Weiterbildungsgang. Denkbar, wenn auch schwer prak-

Trauma Berufskrankh 2010 - 12[Suppl 2]:153-156 DOI 10.1007/s10039-010-1647-2

(c) Springer-Verlag 2010

V. Bühren

Heilverfahren der DGUV. Heilverfahren aus der Sicht der Fachgesellschaften

\section{Zusammenfassung}

Aus Sicht der wissenschaftlichen Gesellschaften besteht inzwischen Konsens mit der Deutschen Gesetzlichen Unfallversicherung (DGUV) über eine zukünftige Modularität in den Anforderungen an den Durchgangsarzt (D-Arzt). Die Basis wird vom Facharzt für Orthopädie und Unfallchirurgie gebildet, ergänzt durch ein weiteres Jahr an einem zum Verletzungsartenverfahren (VAV) zugelassenen Haus nach der Facharztprüfung. Hinzu kommen spezielle Kurse zu Themen wie Heilverfahrenssteuerung, Gutachten und Rehabilitation. Für den operierenden D-Arzt bleibt es bei der Regelung des Facharztes für Orthopädie und Unfallchirurgie mit anschließender erworbener Zusatzweiterbildung Spezielle Unfallchirurgie. Für den D-Arzt im VAV werden zusätzlich 3 Jahre an einem zum VAV zugelassenen Haus gefordert. Ein zusätzliches Traumanetz SGB (Sozialgesetzbuch) VII wird in Synergie zum schon bestehenden Traumanetzwerk ${ }^{\mathrm{D}}$ der Deutschen Gesellschaft für Unfallchirurgie ausgebildet werden. Prinzipiell werden regionale Traumazentren über eine Zulassung zum VAV verfügen und umgekehrt. Die Häuser mit Zertifizierung als überregionales Traumazentrum sind grundsätzlich geeignet, auch die Rolle eines Traumazentrums im SGB VII-Netz zu übernehmen.

\section{Schlüsselwörter}

Deutsche Gesetzliche Unfallversicherung . Heilverfahren - Traumanetzwerk ${ }^{\mathrm{D}}$. Verletzungsartenverfahren - Durchgangsarztverfahren

\section{Medical care under German statutory accident insurance. Perspective of medical associations}

\section{Abstract}

From the perspective of German scientific societies and associations, a consensus has now been reached with the German statutory accident insurance on the modularity in requirements on authorised medical examiners in the future. The basis is formed by the specialty in orthopedics and trauma surgery, complemented by an additional year (following specialist medical examination) at a center authorized in injury typing procedures. Additional courses on medical care management, expert opinions and rehabilitation are also included. For medical examiners who also operate, the regulations for specialists in orthopedics and trauma surgery, followed by additional further training in special trauma surgery, remain valid. Three additional years at an authorized injury-typing-procedure center are required for medical exam- iners specialized in injury typing procedures, An additional trauma network SGB (German Social Code) VII will be developed in synergy with the Traumanetzwerk ${ }^{\mathrm{D}}$ (trauma network) which already exists under the auspices of the German Society of Trauma Surgery. In general, regional trauma centers will be authorized to perform injury typing procedures and vice versa. Centers certified as national trauma centers are in principle able to assume the role of a trauma center in the SGB VII network.

\section{Keywords}

German statutory accident insurance . Medical care · Trauma network · Injury typing procedures · Authorized medical examination procedures 
tikabel, wäre eine separate Anerkennung durch die DGUV.

\section{Synergien zwischen den Traumanetzwerken}

Das Traumanetzwerk ${ }^{\mathrm{D}}$ der Deutschen Gesellschaft für Unfallchirurgie sieht eine Dreistufigkeit mit lokalen, regionalen oder überregionalen Traumazentren vor.

Das Verletzungsartenverfahren der DGUV erfordert in der Regel eine 2-jährige Weiterbildungsbefugnis in der Speziellen Unfallchirurgie und sieht in Analogie $\mathrm{zu}$ den früher geltenden Regelungen eine quantitative Mindestgröße von 50 Fällen pro Jahr im Krankenhaus als untere Grenze. Erfahrungsgemäß sind zum Erreichen dieser Anforderungen eine Größe der Abteilung über 40 Betten sowie die Kapazität von 2 OP durchgehend über das Jahr erforderlich.

Werden diese Leistungsparameter auf das Traumanetzwerk ${ }^{\mathrm{D}}$ der DGU projiziert, zeigt sich eine erhebliche Übereinstimmung mit der Definition des regionalen Traumazentrums. Letztendlich sind die gegenläufigen Aussagen berechtigt, dass ein regionales Traumazentrum regelhaft am Verletzungsartenverfahren beteiligt sein soll, ein am Verletzungsartenverfahren beteiligtes Haus sollte regelhaft als regionales Traumazentrum zertifiziert sein.

Für die überregionalen Traumazentren ist eine höhere Versorgungskapazität erforderlich, als Faustregel kann hier eine Abteilungsgröße von 70 Betten und mehr definiert werden, es sollten regelhaft und durchgehend 3 OP zur Verfügung stehen. Derartig im Traumanetzwerk ${ }^{\mathrm{D}}$ der DGU qualifizierte Kliniken könnten künftig die Basis für das geplante Traumanetz SGB VII der DGUV bilden.

Angesichts sinkender Unfallzahlen, einer Konzentrierung der unfallchirurgischen Versorgung auf Zentren und der Strukturierung innerhalb des Fachgebietes Orthopädie und Unfallchirurgie ist zu erwarten, dass sich die Zahl der am Verletzungsartenverfahren beteiligten Kliniken in den nächsten Jahren deutlich reduzieren wird. Die Schätzungen gehen von einer Halbierung der bisher etwa 600 beteiligten Kliniken aus. Von den verbleibenden etwa 300 Häusern könnten et- wa $20 \%$, somit 60 Kliniken, auf dem Niveau des SGB VII-Traumazentrums arbeiten. Leistungszahlen, Qualitätsanforderungen und die Honorierung für derartige Zentren im Rahmen der DGUVHeilverfahren wird Gegenstand einer anhaltenden Diskussion mit den wissenschaftlichen Fachgesellschaften der nächsten Jahre sein.

\section{Korrespondenzadresse}

\section{Prof. Dr. V. Bühren}

BG-Unfallklinik Murnau,

Prof.-Küntscher-Straße 8, 82418 Murnau

buehren@bgu-murnau.de

Interessenkonflikt. Der korrespondierende Autor gibt an, dass kein Interessenkonflikt besteht. 